\title{
Beneficial Effects of Grape Resveratrol on Serum Adiponectin and Inflammation: Clinical Trial in Patients with Stable Coronary Artery Disease
}

\author{
Editorial to: "Grape Resveratrol Increases Serum Adiponectin and Downregulates \\ Inflammatory Genes in Peripheral Blood Mononuclear Cells: A Triple-Blind, \\ Placebo-Controlled, One-Year Clinical Trial in Patients with Stable Coronary Artery \\ Disease" by J. Tomé-Carneiro et al.
}

\author{
Subhash K. Das • Vaibhav B. Patel • Gavin Y. Oudit
}

Published online: 6 January 2013

(C) Springer Science+Business Media New York 2013

Coronary artery disease (CAD) is a leading cause of heart failure on a world-wide basis [1-3]. The epidemic proportion of CAD is increasing worldwide irrespective of gender $[3,4]$. Coronary artery disease is associated with lower oxygen supply and demand ratio due to abnormalities of the coronary vasculature. The coronary arteries supply oxygenated blood to the heart muscle and due to the process of atherosclerosis leading to plaque rupture and acute coronary syndrome, myocardial blood supply is compromised leading to myocardial ischemia/infarction and heart failure $[5,6]$. Many clinicians and cardiovascular researchers recognize the clinical as well as molecular pathophysiological basis of coronary artery disease. There are many research groups actively searching the intervention strategies to prevent CAD. Many of these studies confirmed that CAD can be favorably prevented by lifestyle modifications (dietary

S. K. Das • V. B. Patel • G. Y. Oudit

Division of Cardiology, Department of Medicine,

University of Alberta, Edmonton, Canada

S. K. Das $\cdot$ V. B. Patel $\cdot$ G. Y. Oudit

Mazankowski Alberta Heart Institute,

University of Alberta, Edmonton, Canada

G. Y. Oudit

Department of Physiology, University of Alberta,

Edmonton, Canada

G. Y. Oudit $(\bowtie)$

Division of Cardiology, Department of Medicine,

Mazankowski Alberta Heart Institute, University of Alberta,

Edmonton, Alberta T6G 2S2, Canada

e-mail: gavin.oudit@ualberta.ca control, exercise and smoking cessation) and pharmacotherapy [7]. However current pharmacotherapies which include anti-platelet drugs (aspirin, clopidogrel and prasugrel), betablockers, statin therapy and ACE inhibitors, are suboptimal $[8,9]$. Resveratrol is a polyphenolic flavonoid with phytoalexin properties and has several pleotropic effects on cardiovascular function such as anti-oxidant and antiinflammatory effects and metabolic modulation (Fig. 1) [10-14]. However, these studies were performed in animal models and clinical evidence of the therapeutic effectiveness of resveratrol in humans is limited.

In this issue of Cardiovascular Drugs and Therapy, Tomé-Carneiro et al. investigate and analyze the beneficial pleotropic effects of 1 year dietary intervention with resveratrol containing grape extract (U.V treated grapes) on inflammatory as well as fibrinolytic status in patients with stable coronary artery disease [15]. Naturally the content of resveratrol in dietary sources is very low and the consumption of these natural food items (wine and grapes) does not exert beneficial effects in the prevention of many cardiovascular complications. As such Tomé-Carneiro et al. used a novel resveratrol rich grape extract (GE-RES) obtained from U.V treated grapes (GE-RSE) in dietary intervention of CAD patents. Resveratrol is a potent therapeutical phytochemical with cardioprotective effects [10]. It targets and modulates pathological death signals and can protect patients by preventing several cardiovascular risk factors from causing endothelial dysfunction, inflammation, atherosclerotic plaque formation and dyslipidemia [1]. Inflammation and atherogenesis are the initial manifestations in coronary artery disease, which is associated with focal 


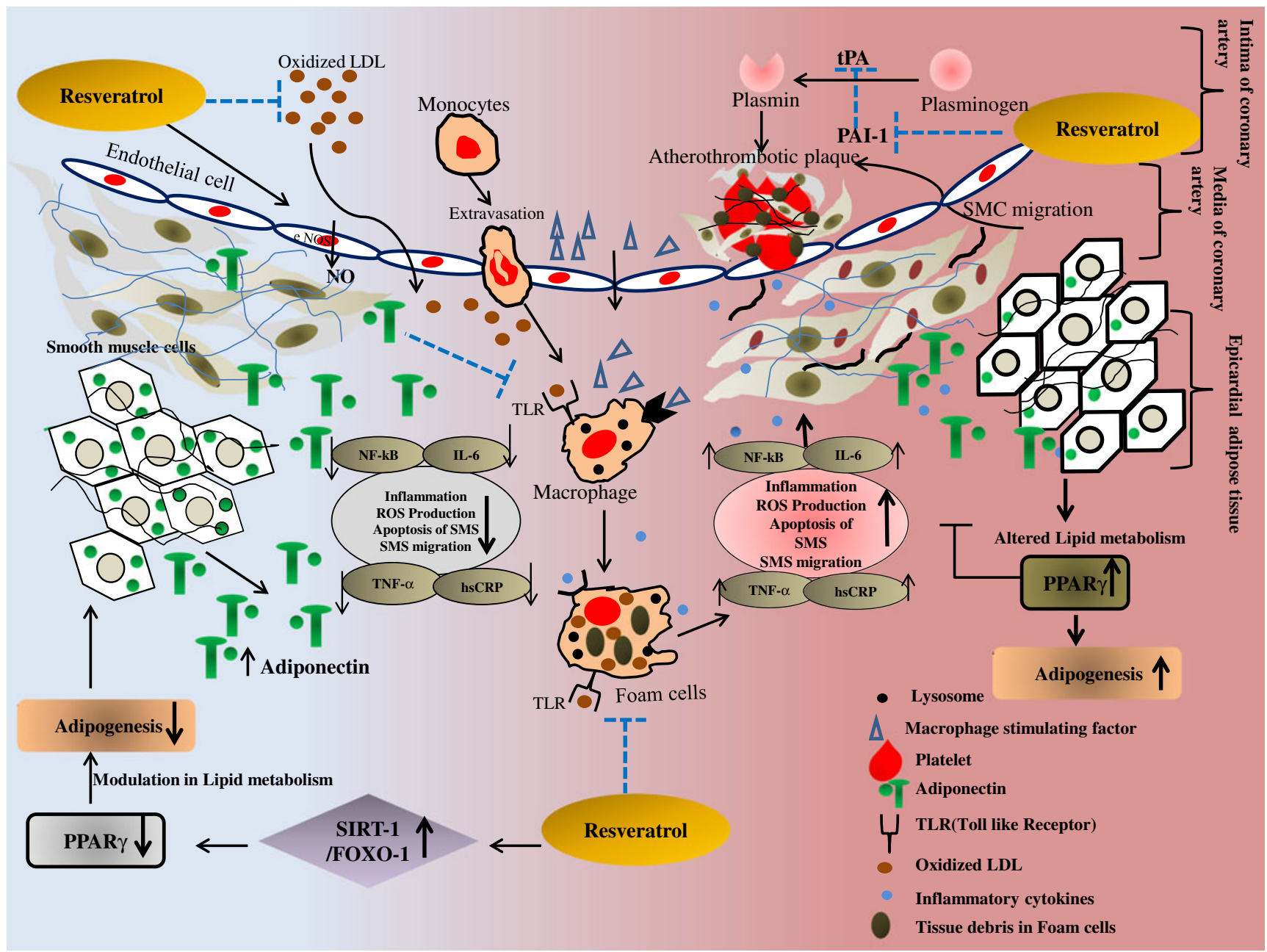

Fig. 1 Altered lipid metabolism reduces adiponectin and upregulates inflammatory signals while resveratrol dietary intervention prevents inflammation, altered lipid metabolism, and stabilizes adiponectin levels through SIRT-I/FOXO1 pathway. Pathological pathways showing altered lipid metabolism and excess adipogenesis in epicardial adipose tissue associated with downregulation of adiponectin. Oxidized LDL stimulates macrophages leading to the accumulation of excess lipids and the formation of foam cells which in turn secrete various inflammatory cytokines and also activate apoptotic signals and ROS production. Migratory smooth muscle cells, platelets, fibrins and oxidizedLDL get accumulated into the inner wall of intima (luminal side of endothelium) of coronary artery and form atherothrombotic plaques.
Resveratrol dietary intervention activates SIRT-1/FOXO1 transcription factors which modulate PPAR $\gamma$ and adipogenesis in EAT leading to decreased adipogenesis stimulates upregulation of adiponectin, which along with its anti-inflammatory action results in a plethora of beneficial effects on the coronary arteries. LDL=Low density lipoprotein; tPA=tissue plasminogen activator; eNOS=endothelial nitric oxide synthase; PAI-1=Plasminogen activator inhibitor-1; PPAR $\gamma=$ Peroxisome proliferator-activated receptor $-\gamma$; SIRT- $1=$ Sirtuin- 1 ; FOXO1 $=$ Forkhead box protein $\mathrm{O} 1$; TNF $\alpha=$ Tumor necrosis factor $\alpha$; hsCRP=high sensitive C-reactive protein; IL-6=Interleukin-6; NF-kB=Nuclear factor kappa $\mathrm{B}$; SMC=Smooth muscle cell; ROS=Reactive oxygen species; TLR=Toll-like receptor activation of endothelial cells via phospholipids, which are produced by enzymatic oxidation of LDL inside and outside of the coronary blood vessel (Fig. 1) [1, 16]. Endothelial activation recruits at first platelets and expresses several types of leukocyte adhesion molecules favoring blood cell adhesion and migration into sub-endothelial spaces [1]. When these monocytes are stimulated by specific cytokines, macrophage stimulating factors (MSF), secreted from tunica intima cells of the vessels, differentiate into macrophages and later into foam cells. Foam cells release pro-inflammatory cytokines, proteases, and cytotoxic superoxide, nitrogen free radicals and activate the inflammatory process along with atherothrombus formation which also in association with elevated systemic levels of inflammatory cytokines (IL-6, IL-7, IL-8, high sensitivity C-reactive protein and $\mathrm{SCD} 40$ ) in the plasma $[16,17]$. The balance between inflammatory and antiinflammatory responses plays an important role in atherothrombus formation and disease progression. The epicardial adipose tissue (EAT) of coronary arteries also directly regulates the coronary vascular function and homeostasis because of its anatomical proximity by secreting various cytokines collectively called adipokines such as adiponectin, leptin, 
resistin, apelin and plasminogen activator inhibitor (PAI-1) $[18,19]$. Recent studies on epicardial adipose tissues confirmed the role of various proatherogenic/pro-inflammatory and atheroprotective/anti-inflammatory cytokines in human coronary artery disease progression [20]. Adiponectin as well as tissue plasminogen inhibitor are two important adipokines produced by epicardial adipose tissues and show inverse relationship in many cardiovascular as well as metabolic disorders [21].

Adiponectin is a clinically interesting, important serum adipocytokine, with 224 amino acid residues. It positively regulates glucose and lipid metabolism, and has multiple anti-inflammatory effects [22]. Epidemiological studies confirmed the low circulatory levels of adiponectin in CAD [23, 24]. Adiponectin participates in the in vivo clearance of apoptotic cellular bodies and blebs of dying cells by binding them, leading to phagocytosis by monocyte derived macrophages, thereby minimizing tissue injury and inflammation $[25,26]$. Adiponectin dependent suppression of inflammatory cytokines is well established in in vitro and in vivo studies [2, 20, 27]. An alteration in epicardial adipocyte metabolism leads to excess adipogenesis and down regulates the adiponectin gene expression [27]. The adipose tissue associated with the epicardial coronary arteries also contains fibroblasts, pre-adipocytes, and $5-10 \%$ of tissue macrophages. Alterations in fat metabolism stimulate excess infiltration of monocyte derived macrophages, leading to accumulation of lipid particles and formation of foam cells [28]. The latter stimulate adipocytes, downregulate their adiponectin expression and upregulate the expression of cytokines such as IL-6, TNF- $\alpha$, PAI-1, angiotensinogen, CRPs and many transcription factors (NF-kB, AP-1, JUN, ATF-2, CREB) [28]. The plasminogen activator inhibitor (PAI-1) is also an adipokine and regulatory protein secreted by platelets, endothelium as well as adipose tissue and participates in the coagulation cascade $[29,30]$. PAI- 1 is a key regulator which determines the endogenous fibrinolysis activity and principle inhibitor of fibrinolysis [31]. Several cardiovascular and metabolic abnormalities are associated with elevated levels of PAI-1, including the upregulation of TNF- $\alpha$, up regulates PAI- 1 and IL-6, activation of NF-kB transcriptional factor, and downregulation of adiponectin expression [31].

Tomé-Carneiro et al. show that resveratrol containing grape extract (GE-RSE-UV treated) increases adiponectin levels and decreases hsCRP and PAI-1 levels in plasma, in addition to downregulation of pro-inflammatory gene expression of PBMCs in stable CAD patients. These results clearly illustrate thast GE-RSE dietary intervention leads to a drastic improvement in circulating biomarker levels and are consistent with previous findings showing that resveratrol mediates SIRT1/FOXO1 dependent upregulation of adiponectin and downregulation of PPAR $\gamma[32,33]$. SIRT-
$1 / \mathrm{FOXO} 1$ is one the major signaling pathways activated by resveratrol. Activation of SIRT-1(Sirtuin-1) and FOXO1 (Forkhead transcription factor) by resveratrol is a wellestablished signaling pathway. SIRT-1 catalyzes NAD ${ }^{+}$-dependent protein deacetylation, which is a very crucial metabolic modulation catalytic reaction to prevent obesity and related metabolic disorders [34]. FOXO1 activation inhibits adipogenesis [35]. The nuclear peroxisome proliferatoractivated receptor $\gamma$ (PPAR $\gamma$ ) plays a central role in adipogenesis and fat tissue development. Resveratrol is a negative regulator of the nuclear peroxisome proliferator-activated receptor gamma (PPAR $\gamma$ ), which is known to play a central role in adipogenesis and vascular disease [34].

Activation of inflammation and pro-inflammatory gene expression in PBMCs is also associated with pathogenesis of CAD. The study by Tomé-Carneiro et al. also shows that resveratrol reduces inflammation and downregulates the pro-inflammatory gene expression in PBMCs. Increased production of TNF- $\alpha$ directly induces synthesis and excess release of PAI-1 in epicardial adipose tissue and increases plasma PAI-1 associated with decreased fibrin clearance and enhanced thrombosis [31]. Acute and chronic stimulation by TNF- $\alpha$ activates PKC, PI3K, MAPK and NF-kB signaling pathways leading to induction of PAI-1 expression [31]. Resveratrol can also inhibit TNF- $\alpha$ induced PAI-1 formation and the activation of the NF-kB pathway [36]. Macrophages are important inflammatory cells involved in the initiation of inflammation process and pathogenesis of many diseases including CAD. Macrophage infiltration is also higher in epicardial adipose tissue and coronary arteries of $\mathrm{CAD}$ patients. The amplification of the inflammatory transcription factors in PBMCs leads to the release of inflammatory cytokines [37]. Six inflammatory transcription factors were examined by Tomé-Carneiro et al., including Kruppel like factor2 (KLF2), nuclear factor kappa-B (NF$\mathrm{KB})$, activator protein-1(AP-1), c-Jun, activating transcription factor 2 (ATF-2), and CREB-binding protein. Upon resveratrol intervention the gene expression pattern of these genes are drastically modulated. These authors have previously shown a clear benefit of UV treated grape extract in stable CAD patients, by increasing the level of pleotropic adipokine, adiponectin, and decreasing PAI-1, IL-6, and TNF- $\alpha$ inflammatory cytokines [36].

Plants and plant products have one or more medicinal values, and medicinal plants have been used as traditional treatments of many life threatening diseases for thousands of years all over the world. Vitis vinifera (Grape plant) contains resveratrol phytoallexin as a defensive molecule. The popular 'French paradox' is well-known, which states that the low CVD mortality rate in French population is linked to the consumption of red wine [14]. As we move ahead, the pharmacokinetics such as the therapeutic levels of plasma and tissue resveratrol in response to dietary intervention as 
well as the pharmacodynamics of resveratrol have to be better understood. Importantly, we need to better define the absolute resveratrol concentration that is effective for CAD treatment, and how it will be a better therapeutic agent compared to other currently available therapies for CAD.

Acknowledgements We acknowledge the financial support of an operating grant from the Heart and Stroke Foundation of Canada.

\section{References}

1. Hansson GK. Inflammation, atherosclerosis, and coronary artery disease. N Engl J Med. 2005;352:1685-95.

2. Zhou Y, Wei Y, Wang L, et al. Decreased adiponectin and increased inflammation expression in epicardial adipose tissue in coronary artery disease. Cardiovasc Diabetol. 2011;10:2.

3. Nabel EG, Braunwald E. A tale of coronary artery disease and myocardial infarction. N Engl J Med. 2012;366:54-63.

4. Canoy D. Distribution of body fat and risk of coronary heart disease in men and women. Curr Opin Cardiol. 2008;23:591-8.

5. Libby P. Inflammation in atherosclerosis. Nature. 2002;420:86874.

6. Hallenbeck JM, Hansson GK, Becker KJ. Immunology of ischemic vascular disease: plaque to attack. Trends Immunol. 2005;26:550-6.

7. Capewell S, Morrison CE, McMurray JJ. Contribution of modern cardiovascular treatment and risk factor changes to the decline in coronary heart disease mortality in Scotland between 1975 and 1994. Heart. 1999;81:380-6.

8. Veillard NR, Mach F. Statins: the new aspirin? Cell Mol Life Sci. 2002;59:1771-86.

9. Ozasa N, Morimoto T, Bao B et al. beta-blocker use in patients after percutaneous coronary interventions: One size fits all? Worse outcomes in patients without myocardial infarction or heart failure. Int J Cardiol 2012.

10. Baur JA, Pearson KJ, Price NL, et al. Resveratrol improves health and survival of mice on a high-calorie diet. Nature. 2006;444:33742.

11. Chung JH, Manganiello V, Dyck JR. Resveratrol as a calorie restriction mimetic: therapeutic implications. Trends Cell Biol. 2012;22:546-54.

12. Dolinsky VW, Chan AY, Robillard Frayne I, Light PE, Des Rosiers C, Dyck JR. Resveratrol prevents the prohypertrophic effects of oxidative stress on LKB1. Circulation. 2009;119:1643-52.

13. Brisdelli F, D'Andrea G, Bozzi A. Resveratrol: a natural polyphenol with multiple chemopreventive properties. Curr Drug Metab. 2009; 10:530-46.

14. Catalgol B, Batirel S, Taga Y, Ozer NK. Resveratrol: French paradox revisited. Front Pharmacol. 2012;3:141.

15. Tomé-Carneiro J, Gonzalvez M, Larrosa M, et al. Grape resveratrol increases serum adiponectin and downregulates inflammatory genes in peripheral blood mononuclear cells: a triple-blind, placebo-controlled, one-year clinical trial in patients with stable coronary artery disease. Cardiovasc Drugs Ther 2013;27: this issue

16. Berg AH, Scherer PE. Adipose tissue, inflammation, and cardiovascular disease. Circ Res. 2005;96:939-49.

17. Veillard NR, Kwak B, Pelli G, et al. Antagonism of RANTES receptors reduces atherosclerotic plaque formation in mice. Circ Res. 2004;94:253-61.
18. Tilg H, Moschen AR. Adipocytokines: mediators linking adipose tissue, inflammation and immunity. Nat Rev Immunol. 2006;6:772-83.

19. Iacobellis G, Corradi D, Sharma AM. Epicardial adipose tissue: anatomic, biomolecular and clinical relationships with the heart. Nat Clin Pract Cardiovasc Med. 2005;2:536-43.

20. Karastergiou K, Evans I, Ogston N, et al. Epicardial adipokines in obesity and coronary artery disease induce atherogenic changes in monocytes and endothelial cells. Arterioscler Thromb Vasc Biol. 2010;30:1340-6.

21. Bilgili S, Celebiler AC, Dogan A, Karaca B. Inverse relationship between adiponectin and plasminogen activator inhibitor-1 in metabolic syndrome patients. Endocr Regul. 2008;42:63-8.

22. Zhu W, Cheng KK, Vanhoutte PM, Lam KS, Xu A. Vascular effects of adiponectin: molecular mechanisms and potential therapeutic intervention. Clin Sci (Lond). 2008;114:361-74.

23. Nakamura Y, Shimada K, Fukuda D, et al. Implications of plasma concentrations of adiponectin in patients with coronary artery disease. Heart. 2004;90:528-33.

24. Antoniades C, Antonopoulos AS, Tousoulis D, Stefanadis C. Adiponectin: from obesity to cardiovascular disease. Obes Rev. 2009;10:269-79.

25. Gupta G, Surolia A. Collectins: sentinels of innate immunity. Bioessays. 2007;29:452-64.

26. Takemura Y, Ouchi N, Shibata R, et al. Adiponectin modulates inflammatory reactions via calreticulin receptor-dependent clearance of early apoptotic bodies. J Clin Invest. 2007;117:375-86.

27. Liu M, Liu F. Up- and down-regulation of adiponectin expression and multimerization: mechanisms and therapeutic implication. Biochimie. 2012;94:2126-30.

28. Yudkin JS, Kumari M, Humphries SE, Mohamed-Ali V. Inflammation, obesity, stress and coronary heart disease: is interleukin-6 the link? Atherosclerosis. 2000;148:209-14.

29. Samad F, Loskutoff DJ. Tissue distribution and regulation of plasminogen activator inhibitor-1 in obese mice. Mol Med. 1996;2:568-82.

30. Alessi MC, Peiretti F, Morange P, Henry M, Nalbone G, JuhanVague I. Production of plasminogen activator inhibitor 1 by human adipose tissue: possible link between visceral fat accumulation and vascular disease. Diabetes. 1997;46:860-7.

31. Pandey M, Loskutoff DJ, Samad F. Molecular mechanisms of tumor necrosis factor-alpha-mediated plasminogen activator inhibitor-1 expression in adipocytes. FASEB J. 2005;19:1317-9.

32. Picard F, Kurtev M, Chung N, et al. Sirt1 promotes fat mobilization in white adipocytes by repressing PPAR-gamma. Nature. 2004;429:771-6.

33. Subauste AR, Burant CF. Role of FoxO1 in FFA-induced oxidative stress in adipocytes. Am J Physiol Endocrinol Metab. 2007;293: E159-64.

34. Floyd ZE, Wang ZQ, Kilroy G, Cefalu WT. Modulation of peroxisome proliferator-activated receptor gamma stability and transcriptional activity in adipocytes by resveratrol. Metabolism. 2008;57:S32-8.

35. Wang F, Tong Q. SIRT2 suppresses adipocyte differentiation by deacetylating FOXO1 and enhancing FOXO1's repressive interaction with PPARgamma. Mol Biol Cell. 2009;20:801-8.

36. Tome-Carneiro J, Gonzalvez M, Larrosa M, et al. One-year consumption of a grape nutraceutical containing resveratrol improves the inflammatory and fibrinolytic status of patients in primary prevention of cardiovascular disease. Am J Cardiol. 2012;110:356-63.

37. Coenen KR, Gruen ML, Lee-Young RS, Puglisi MJ, Wasserman $\mathrm{DH}$, Hasty AH. Impact of macrophage toll-like receptor 4 deficiency on macrophage infiltration into adipose tissue and the artery wall in mice. Diabetologia. 2009;52:318-28. 